\title{
On best practices: The Institute of Medicine scheme for developing, validating, and demonstrating clinical utility of omics-based diagnostic and predictive tests
}

\author{
Gilbert S. Omenn ${ }^{1,2}$ \\ ${ }^{1}$ Departments of Computational Medicine and Bioinformatics, Internal Medicine, and Human Genetics, School of \\ Public Health, University of Michigan, Ann Arbor, MI, USA \\ ${ }^{2}$ Institute for Systems Biology, Seattle, WA, USA
}

\begin{abstract}
The Institute of Medicine of the US National Academy of Sciences issued a major report in 2012 that provides important guidance for the proteomics community for credible and transparent development of all kinds of omics-based diagnostic and prognostic tests. The key elements are summarized here to provide a compass that can guide the studies emerging from many laboratories as reported in this special issue of Proteomics Clinical Applications on "Diagnostic Proteomics."
\end{abstract}

\section{Keywords:}

Diagnostics / Institute of Medicine / Omics / Tests

\section{Introduction}

Molecular signatures are becoming useful biomarkers for diagnosis, prognosis, choice of therapy, response to therapy, and recurrence of disease. Proteomics, especially with the new SRM-targeted proteomics approaches, is in the center of this work. In fact, integration of multiple omics approaches (genomics, epigenomics, transcriptomics, miR-omics, proteomics, metabolomics) is likely to be the most comprehensive and productive route to "personalized" or "precision medicine" [1]. Integration is a key feature of our scheme for linking from genome to phenome, or phenome to genome [2]; see Fig. 1.

Omics research generates complex high-dimensional data. Often there are measurements of far more variables per sample than the total number of biological samples used to generate the dataset. These data require a computational model or algorithm to distinguish a health-related characteristic of clinical significance in one group (patients) compared with another (controls/normal) or in two different subgroups of patients with the same general diagnosis.

Correspondence: Dr. Gilbert S. Omenn, Department of Computational Medicine and Bioinformatics, University of Michigan, Ann Arbor, MI 48109-2218, USA

E-mail: gomenn@umich.edu

Fax: +1-734-615-6553

Abbreviations: CLIA, Clinical Laboratory Improvement Amendments; FDA, Food and Drug Administration; IDE, investigational device exemption; IRB, Institutional Review Board
Received: May 31, 2013

Revised: August 26, 2013

Accepted: August 28, 2013
As emphasized in the 2012 report "Evolution of Translational Omics: Lessons Learned and Path Forward," [3] high-dimensional data are prone to "overfitting." This phenomenon refers to results that cannot be replicated in an independent set of samples, a huge problem in the literature of disease biomarkers. A computational model created in the discovery phase of research on a certain set of samples may be less accurate or useless when applied to another set of samples. A standardized, carefully executed series of studies is required to achieve a validated clinical test suitable for evaluation in a clinical setting, especially for making decisions to improve outcomes from care of the patient.

Omics-based tests differ from common single analyte tests in many respects. Unlike a specific assay for LDL receptor or a hemoglobin variant or a particular enzyme, where a sound biological rationale links the assay to the disease process, global analysis of nearly all the genes or hundreds or thousands of proteins in "unbiased discovery studies" rests on statistical and informatics methods for interpretation.

\section{The importance of transparent, fully annotated data sharing}

The complexity of omics research puts a premium on data provenance and sharing of both data and the details of the computational model. Proteomics researchers have lagged far behind genomics researchers in promptly committing their data and metadata to publicly accessible data repositories.

Colour Online: See the article online to view Figs. 1 and 2 in colour. 


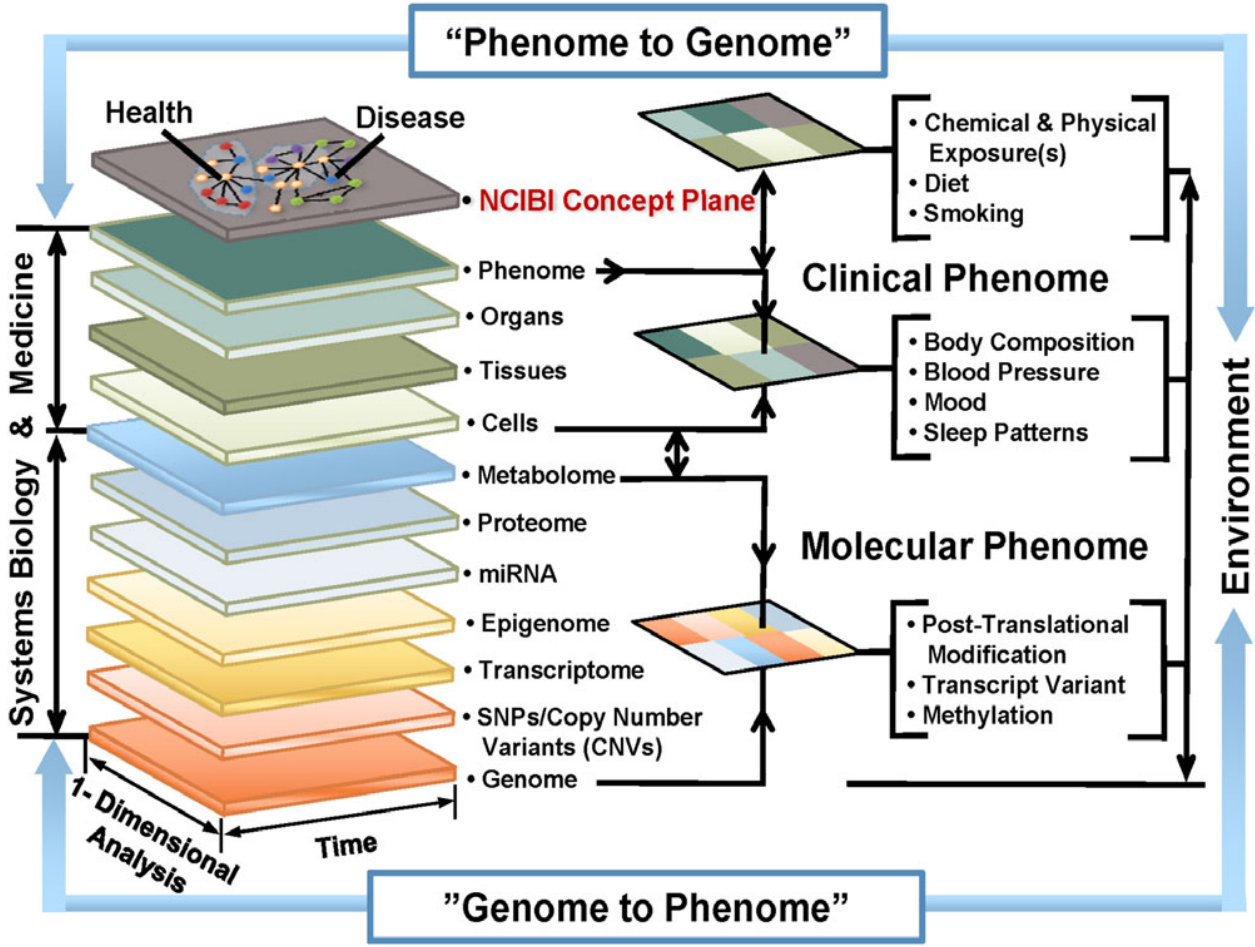

Figure 1. Scheme showing multidimensional omics analyses, physiological and clinical measures, and environmental, behavioral, and social influences on health and disease, linking genome and phenome (phenotypes). Source: [2] p.51.
Without access to the data and classifier for independent confirmation, it is not feasible for others to replicate and verify the results.

The HUPO Human Proteome Project (www.thehpp.org) has committed that the participating investigator teams will submit their datasets through the new ProteomeXchange mechanism (Vizcaíno et al., Nat. Biotechnol., in press) into the curated PRIDE repository at the European Bioinformatics Institute and into Peptide Atlas at the Institute for Systems Biology for standardized reanalysis using the TransProteomicPipeline. GPMdb also combines and reanalyzes datasets, using X!Tandem. Targeted MRM/SRM proteomics data can now be submitted to SRM Atlas using PASSEL [4].

\section{The IOM scheme for development of omics-based tests}

The development of omics-based multianalyte tests has been much slower than expected. A major reason is the lack of a widely agreed-upon process for translation of omics discoveries into clinical omics-based tests. Detailed case studies of six clinically useful multianalyte tests are presented in Appendix A of the 2012 IOM report [3]: Oncotype DX and MammaPrint to guide decisions on whether patients treated for breast cancer are at sufficient risk of recurrence and metastases that they should have toxic adjuvant chemotherapy to reduce the risk of metastases; Tissue of Origin for cancers of unknown site of origin; OVA1, a set of five abundant proteins, to guide the choice of a gynecologic surgeon instead of a general surgeon for women with known pelvic mass that might be ovarian cancer or other, often benign, tumor; AlloMap to monitor cardiac transplant patients for early immune rejection; and Corus CAD to screen patients with symptoms suggestive of myocardial ischemia in the clinic or emergency ward.

A widely reported failure of a set of highly touted omicsbased tests to direct the choice of the most effective cancer chemotherapy agent for individual patients led to the request by the National Cancer Institute and Duke University for the Institute of Medicine to review this emerging field and provide guidance on best practices, or "how to do it right" [3].

This guidance will be important for proteomics-based biomarker test development, the subject of this special issue. The IOM report clarifies the responsibilities of all the investigators/co-authors, as well as of institutional review bodies, funding agencies, journal editors and reviewers, and the Food and Drug Administration (FDA) regulatory process.

Figure 2 lays out the three-phase process for omics-based tests, with development and validation of the test in the first two phases (first stage) and evaluation of the clinical utility in a third phase (second stage). There is a bright line to be passed before entering the assessment of clinical utility (second stage).

\subsection{Discovery phase}

The product of the discovery phase is a fully described laboratory assay and a fully described, locked-down computational procedure; before validation, it is important to confirm the initial findings with an independent set of samples in the 


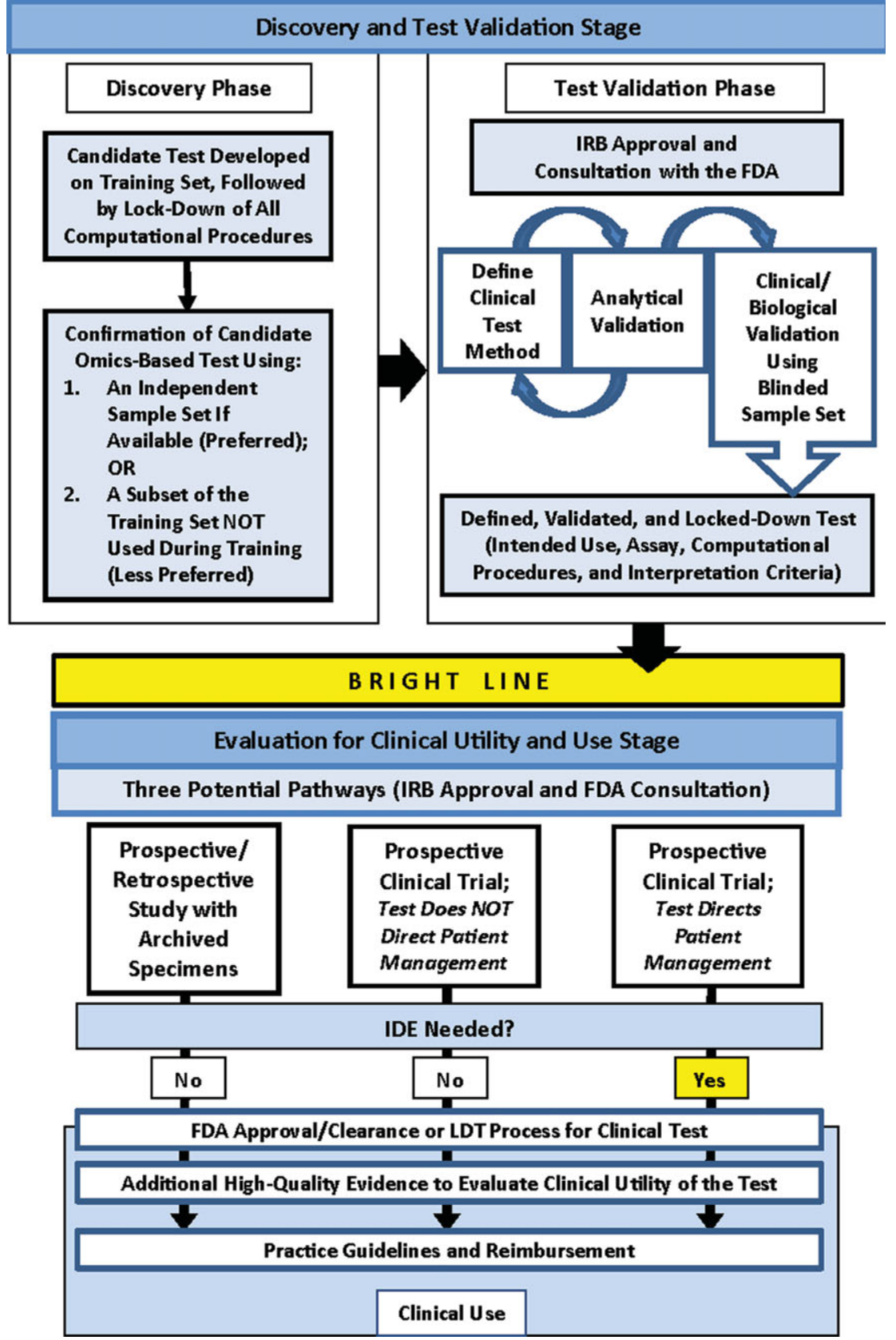

Figure 2. Omics-based test development process, in three phases: discovery and confirmation, test validation in a clinical laboratory, and evaluation for clinical utility and clinical use. Source: [3] p.6. same laboratory with the same staff and the same technology platform. A common error in the discovery phase is to mingle the "training set" of samples and the "test set of samples," or to combine the two after initial separate analysis, hoping to enhance the AUC (area under the curve in a plot of specificity vs. sensitivity). Such mingling should never be done. It is an inexcusable error to know which samples are from patients and which from controls, breaking the "blinded status" of the analysis.

For a long time, it was standard practice in research to repeat an experiment before rushing to publish or license; now it is too often the case that a quite preliminary discovery phase result is published without replication. In some cases, there is a scheme for this preliminary result to be "handed off" to a different lab for validation, as in the NCI Early Detection Research Network. I highly recommend the IOM guidance that the original laboratory should perform a confirmation step before the test proceeds for analytical validation.

\subsection{Test validation phase}

The test validation phase moves the in vitro multianalyte assay from the research lab to a CLIA-certified (where CLIA 
is Clinical Laboratory Improvement Amendments) clinical pathology laboratory for high throughput, rigorous calibration and quality assurance, and standardized practice. One must note that CLIA certifies the laboratory, not the specific test. Often the technology platform for the multianalyte test is changed, for example, from MS to ELISA. This change requires a full repeat cycle of the discovery phase training and test set analyses with independent blinded specimens after the new assay and the new computational procedures are locked-down. Moreover, it is important that the specimen sources and preanalytical procedures be matched to the circumstances expected with the intended clinical use of the test. The IOM report insists that the assay details and the algorithm and code for the classifier be made public.

It is wise to seek advice from the FDA at this stage and to determine whether the test development will lead to a submission to FDA for risk-based review and approval of the test as an in vitro diagnostic multianalyte assay or the test will be made available without FDA approval in a single reference lab as a lab-directed test.

\subsection{Evaluation for clinical utility}

The evaluation for clinical utility has three potential pathways, which may be alternatives or may be sequential, depending on the intended use. All of these studies utilize patient specimens or involve patients directly, so full human subjects review by the Institutional Review Board (IRB) and Clinical Protocol Review Committees must be performed and well documented. Clinical trials must be recorded with detailed protocols in www.clinicaltrials.gov. Any potential conflicts of financial interest must be disclosed to the appropriate institutional office and to the IRB for inclusion in the informed consent.

It is highly desirable to seek FDA guidance before undertaking these studies, as noted even for the test validation phase; for the third of these clinical validation pathways, a filing for an investigational device exemption (IDE) is required.

As shown in Fig. 2, the first pathway utilizes archived specimens from an already-completed randomized clinical trial, if such specimens and trial results are available. This prospective-retrospective study design, originally proposed by Simon et al. [5], is an efficient way to evaluate the potential usefulness of the test without putting any patients at risk. Moreover, there is no multiyear wait to see the survival results of the alternative treatments for a prospective study of randomly assigned patient groups.

The second pathway is a prospective assessment of the predictive test with random assignment of patients; the test results have no influence on the therapy given the patients. Afterward, the test results permit comparison of treatment outcomes with the alternative drugs.

The third pathway is the highest use of the predictive omics test: the assignment of patients to specific alternative therapies based on the prestudy results of the test. This path- way requires the risk-based FDA IDE review; the developer still has the option of a laboratory-developed test or an FDA approval.

In the Duke case, the microarray-based molecular signature tests still under development [6] were used prematurely to determine which drug specific patients with lung cancers or breast cancers would receive in three separate clinical trials launched in 2007. The principal investigator, lead author, and 11 and 15 co-authors, respectively, on the two papers failed to recognize the incomplete and error-ridden nature of the published results and chose not to utilize specific criticisms from bioinformaticians at MD Anderson Cancer Center [7] to improve the analyses before launching the three clinical trials and also two companies to perform the laboratory assays and the bioinformatics analyses. Documents prepared by Duke for the IOM Committee review and Appendix B of the IOM report lay out the details and timeline.

\subsection{OVA1: A proteomics-based test with FDA clearance for a narrow intended use}

The following section is a truncated version of the case study in Appendix A of the IOM report, pp. 209-213 [3]. In September 2009, the FDA cleared OVA1 (Vermillion) as the first in vitro diagnostic multivariate index assay $[8,9]$. It is the only proteomics-based test marketed for clinical use, although its clinical version is a multianalyte immunoassay. OVA1 is approved to help identify potential cases of ovarian cancer and guide surgical treatment. OVA1 is the result of collaborative efforts between Johns Hopkins University researchers led by Zhen Zhang and Daniel Chan and scientists at Vermillion including Eric Fung. The intended use of OVA1 is narrowly circumscribed; it is not a screening or a diagnostic test; rather it is intended to guide treatment decisions by "[helping] patients and health care professionals decide what type of surgery should be done and by whom" [9]. OVA1 is used in cases when a patient has an adnexal pelvic mass that is already known to require surgery. It measures serum levels of five plasma proteins-CA 125 II, transthyretin (prealbumin), apolipoprotein A1, beta 2 microglobulin, and transferrin-which change in association with ovarian cancers. The biomarkers included in the OVA1 panel (not including CA 125) were discovered through multicenter proteomic studies [10]. A proprietary algorithm produces a single numerical score between 0 and 10 that indicates the likelihood that a pelvic mass is benign or malignant. A high OVA1 score identifies patients who are likely to benefit from referral to a gynecological oncologist, rather than a general surgeon, for surgery.

Fung recounted his experiences defining the OVA1 assay methodology and the process of obtaining FDA clearance instead of opening a CLIA-certified laboratory without FDA clearance of the specific test [11]. Investigators met FDA before starting clinical trials, during clinical trials, and during the submission process. The FDA helped in framing the test's intended use and the structure of the clinical trials necessary 
to evaluate clinical validity. The investigators initially sought to develop the final algorithm using the SELDI-MS platform that was used to identify the chosen markers. However, they encountered difficulties with the analytic performance of the SELDI methodology (not unlike those issues confronted by Petricoin and Liotta earlier with OVACHECK [3]). They concluded that the SELDI platform did not perform reproducibly enough for routine clinical use. The researchers proceeded to develop immunoassays for the markers that did not already have such assays available. The panel was optimized through several additional clinical trials. An earlier critical decision was to give up on the goal of screening asymptomatic women for ovarian cancers; simulation models demonstrated that even a highly specific test would result in a very low positive predictive value due to a high multiple of false-positive results to true positives [12].

In June 2008, the authors submitted their data to the FDA. Subsequently, the investigators modified the intended use and addressed clinical and analytical questions as a result of discussions with regulatory scientists. One important decision was to generate different cutoff values for premenopausal and postmenopausal women. Data related to assay performance and clinical utility were presented at the 2010 AACR meeting.

Zhang and Chan [13] described critical issues relevant to proteomic-based biomarker discovery and validation, including the inherent complexity of the clinical sample proteome, biological and preanalytical variability, and analytical validity. Practical suggestions for minimizing the effect of potential biases included defining a clear clinical utility from the outset, incorporating multicenter study design, employing analytical randomization, and paying close attention to the factors that contribute to false discovery and model overfitting. Later they shared their insights from the OVA1 development process in a 2010 "lessons learned" commentary [8]. They described three main bridges critical to bringing researchbased biomarker discovery to the level of clinical diagnostics: (i) generation of sufficient and "portable" evidence during preliminary validation studies to support expansion into larger scale validation studies, (ii) careful definition of clinical utility goals that balance broad applicability with feasibility in order to execute clinical trials that will fulfill regulatory requirements, and (iii) selection of assays with the proper analytical performance necessary for clinical use. A space diagram comparing OVA1 and a fictitious screening assay illustrates the tradeoffs between narrowly defined tests (OVA1) and broad-based screening tests, highlighting the consequences of false negatives and false positives and related factors. The authors emphasized the fact that a research assay does not necessarily translate into a clinical assay, relating their switch to an immunoassay format.

The "test” marketed by Quest Diagnostics comprises OvaCalc Software as well as the instruments, assays, and reagents recommended by Vermillion and sold separately from the OvaCalc software. As of 2011, OVA1 had its CE Mark in the European Union and was covered by Medicare, 22 Blue Cross Blue Shield plans, and other private health plans in the United States. On May 15, 2013, Vermilion announced an initiative to migrate OVA1 to a state-of-the-art clinical testing platform commonly available around the world by end of 2014.

\section{The roles of responsible parties in the development and clinical use of omics-based predictive tests}

Here I provide highlights of the recommendations from the IOM report [3].

\subsection{The principal investigator and research team}

There can be no ambiguity that the principal investigator of the discovery laboratory is the person most responsible for the culture of the laboratory, the performance of all members of the laboratory, and transparent and respectful interactions with the institutional oversight groups, ranging from department chairs and deans, to Human Subjects IRBs, Clinical Trials Scientific Protocol Review Committees, and Conflict of Interest Review and Management processes. Potential conflicts of interest must be disclosed to the IRB and potential trial participants. If licensing or company launches are contemplated, the principal investigator/company founder and his or her collaborators must make full disclosures to the Office of Technology Transfer.

It should be obvious that all co-authors on publications and co-investigators on grant proposals and clinical trials protocols must learn enough about the full project and interact sufficiently to have confidence that all required scientific steps and oversight have been completed responsibly. If there are criticisms about any aspect of the work, either internally or externally from journal or funding agency reviewers or colleagues, all co-authors should be informed and contribute to the responses. As noted in the Duke Case Study [3], all authors were held accountable when numerous publications were retracted (in August 2011, Duke reported 14 fully retracted and 13 partially retracted).

\subsection{The research institutions}

In 2002, the National Research Council and Institute of Medicine published "Integrity in Scientific Research: Creating an Environment that Promotes Responsible Conduct [14]. They listed the following practices: "providing leadership in support of responsible conduct of research; encouraging respect for everyone involved in research; promoting productive interactions between trainees and mentors; advocating adherence to the rules regarding all aspects of the conduct 
of research anticipating, revealing, and managing individual and institutional conflicts of interest; arranging timely and thorough inquiries and investigations of allegations of scientific misconduct and applying appropriate administrative sanctions; offering education pertaining to integrity in the conduct of research; and monitoring and evaluating the institutional environment supporting integrity in the conduct of research and using this knowledge for continuous quality improvement."

The IOM 2012 report [3] stated that any institution not capable of managing these complex responsibilities should not be conducting this kind of research. A gap in the institutional procedures appears to be the requirement for an allegation of misconduct before the institution launches an inquiry, let alone an investigation; as Duke leaders testified to the Committee [3], "there were numerous missed signals" about major problems in the conduct of research and oversight of research by the Nevins laboratory.

The IOM recommended that there be a specific IRB member responsible for considering IDE and IND requirements; an institutional official responsible for comprehensive and timely documentation, disclosure, and management of financial and nonfinancial conflicts of interest; an institutional official responsible for establishing and managing a safe system for preventing, reporting, and adjudicating lapses in scientific integrity and risks to patient safety; and an institutional official responsible for responding to inquiries or criticisms about the science being conducted at the institution, including inquiries from journals. Institutions also should be sensitive to the vulnerability of biostatisticians and bioinformaticians employed on grants to demands from powerful principal investigators to find a method that supports the desired findings.

\subsection{Funding agencies, staff, and reviewers}

Funders can and should play a leadership role in supporting a culture of integrity and transparency in science, while providing the resources and guidance to accelerate discovery, translation, and clinical applications. Funding of independent validation of omics-based tests may be justified by the need to make better decisions about advancing biomarker tests through the development path (Fig. 2). The committee recommended that funders require investigators to make all data, metadata, prespecified analysis plans, computer code, and fully specified computational procedures publicly available and readily interpretable at the time of publication or the end of funding. Funders also were advised to support independent repositories for such data and test validation in CLIA-certified labs. They were encouraged to establish lines of communication with other funders that could be useful when serious problems arise in interdependent research.

\subsection{Journals and journal editors}

There is a fundamental problem in the publication business. With extreme emphasis on getting manuscripts into the most prestigious journals with high impact factors, the pressure to produce manuscripts with no warts and no acknowledgement of complications in the findings is distorting the quality and, it seems, the replicability of reported findings. Editors point out that the responsibility for articles rests with the authors, not the unpaid reviewers or editorial staff, especially for articles with complex computational aspects or big datasets.

The IOM Committee recommended that journal editors require authors who submit manuscripts describing clinical evaluations of omics-based tests to register all clinical trials at www.clinicaltrials.gov or an equivalent registry; to make the data, metadata, analysis plans, code, and fully specified computational procedures for a biomarker candidate publicly available in a standard format (e.g. dbGAP); to provide the journal with the sections of the research protocol relevant to the manuscript; to identify each author's role in the various phases of the manuscript; to require the lead and senior authors to attest to the integrity of the study and have the coauthors confirm shared responsibility; and to use checklists for authors to document compliance with appropriate guidelines (e.g. CONSORT, REMARK). Editors were also advised to develop better mechanisms to resolve possible serious errors in publications and to alert the institutional leadership and all authors when a serious question of accuracy or integrity has been raised.

\subsection{The FDA}

The FDA has established increasingly useful lines of communication with academic and industry groups developing multianalyte tests, other devices, and drugs. The Committee recommended that FDA enable investigators and institutions to have a clearer understanding of regulatory responsibilities by completing and finalizing the long-gestating risk-based guidance for omics-based tests. Such a guidance is apparently under final review currently at the Office of Management and Budget. It should clarify the criteria or pros-andcons for bringing an omics-based test to FDA for review and the same for laboratory-developed tests. It might help IRBs, protocol review committees, institutional leaders, and investigators if FDA communicated the IDE requirements for use of omics-based tests in clinical trials.

\subsection{Implications for international drug development}

For many years, there has been a concerted effort toward harmonization of drug development testing and registration policies and guidance involving the United States, Europe, and Japan. As described in a Workshop Summary from the 
Institute of Medicine of the US National Academy of Sciences [15], the International Conference on Harmonization is sponsored by the drug regulatory agencies and pharmaceutical manufacturers of Europe, Japan, and the United States, with the World Health Organization, Health Canada, the European Free Trade Association, and the Federation of Pharmaceutical Manufacturers and Associations as observers. All of these agencies are striving to catch up with the dramatic developments in genomics, proteomics, metabolomics, and other "omics" fields to evaluate diagnostic tests, as well as new drugs. As other countries become major producers of pharmaceuticals, the impetus for harmonization and the need for molecular biology expertise will continue to grow.

European and Japanese developers and marketers of high-profile omics-based clinical tests are or should be well aware of FDA requirements, given their desire to market the test(s) in the United States, just as US companies must learn the policies and requirements for these other regions of the world.

\section{Outlook}

The progress toward "precision medicine" [1] or "predictive, prospective, preventive, and participatory medicine" [16] is accelerating. Multiple highly targeted drugs with companion diagnostic tests are entering clinical trials and clinical practice. The most prominent examples are (i) Gleevec (imatinib) for Philadelphia chromosome-positive chronic myelogenous leukemia with the 9;22 translocation that produces the BCR-ABL kinase; (ii) Herceptin (trastuzumab) for Her2/neu (ERRB2) amplified breast cancers; (iii) Erbitux and Vectibix (cetuximab, panitumamab) for EGF receptors in colorectal cancers; and (iv) Xalkori (crizotinib) for EML-ALK fusion gene/protein in non-small cell lung cancers. In these cases, we have drugs that work for the specific carcinogenic mechanism; with reliable tests, we can match the patients to the specifically indicated drug, with excellent results. Gleevec also works on the same target found in rare gastrointestinal stromal tumors and Herceptin works on nonbreast cancers that have the corresponding ERBB2-amplified mechanism (gastric, colorectal, and rare lung cancers).

At the University of Michigan, the Center for Translational Pathology led by Arul Chinnaiyan has established the MI-Onco-Seq clinical laboratory service to perform RNA-sequencing on tumor specimens to match patients to available therapies and guide new research for much needed additional agents $[17,18]$. We can expect progressive use of multianalyte proteomics based tests in the coming years.

Companion diagnostics for these targeted therapies may be based on individual mutations or on multianalyte assays. More broadly, however, cancer biomarker test development and adoption into clinical use has lagged far behind advances in therapy. Hayes et al. [19] call this record a "vicious cycle" of undervaluation resulting from inconsistent regulatory standards and unreliable reimbursement, as well as insufficient investment in research and development, lax scrutiny of biomarker manuscripts by journals, and inadequate evidence of analytical validity and clinical utility. Co-authors Daniel Hayes, Debra Leonard, and David Ronsohoff were members of the IOM Committee on omics-based predictive tests. They recommend changes in regulation, reimbursement, investment, peer review, and guidelines that require the participation of all the stakeholders. They endorse the IOM framework for development of new omics-based biomarker tests (above). All of these recommendations apply to development of proteomics-based multianalyte tests. They cite an estimate that diagnostics account for $<2 \%$ of total healthcare spending, but influence $60 \%$ of clinical decision-making.

The promise of personalized medicine rests on achieving these objectives, creating a virtuous cycle in which tumor biomarkers are properly valued by all stakeholders.

The author has declared no conflict of interest.

\section{References}

[1] National Research Council, Toward Precision Medicine: Building a Knowledge Network for Biomedical Research and a New Taxonomy Of Disease, National Academies Press, Washington, DC 2011.

[2] Omenn, G. S., in: Ginsgburg, G. S., Willard, H. F. (Eds.), Genomic and Personalized Medicine, Elsevier, New York 2012, pp. 50-59.

[3] Micheel, C. C., Nass, S. J., Omenn, G. S. (Eds.), EvoIution of Translational Omics: Lessons Learned and the Path Forward, National Academies Press, Washington, DC 2012.

[4] Farrah, T., Deutsch, E. W., Kreisberg, R., Sun, Z. et al., PASSEL: the PeptideAtlas SRMexperiment library. Proteomics 2012, $12,1170-1175$.

[5] Simon, R. M., Paik, S., Hayes, D. F., Use of archived specimens in evaluation of prognostic and predictive biomarkers. J. Natl. Cancer Inst. 2009, 101, 1446-1452.

[6] Potti, A., Dressman, H. K., Bild, A., Riedel, R. F. et al., Genomic signatures to guide the use of chemotherapeutics. Nat. Med. 2006, 12, 1294-1300.

[7] Baggerly, K. A., Coombs, K. R., Deriving chemosensitivity from cell lines: forensic bioinformatics and reproducible research in high-throughput biology. Ann. Appl. Stat. 2009, 3, 1309-1334.

[8] Zhang, Z., Chan, D. W., The road from discovery to clinical diagnostics: lessons learned from the first FDA-cleared in vitro diagnostic multivariate index assay of proteomic biomarkers. Cancer Epidemiol. Biomarkers Prev. 2010, 19, 2995-2999.

[9] US Food and Drug Adminstration, FDA clears a test for ovarian cancer. Available at: http://www.fda.gov/NewsEvents/ Newsroom/PressAnnouncements/ucm182057.htm. Retrieved May 5, 2011. Accessed August 23, 2013. 
[10] Zhang, Z., Bast, R. C., Jr., Yu, Y., Li, J. et al., Three biomarkers identified from serum proteomic analysis for the detection of early stage ovarian cancer. Cancer Res. 2004, 64, 5882-5890.

[11] Fung, E. T., A recipe for proteomics diagnostic test development: the OVA1 test, from biomarker discovery to FDA clearance. Clin. Chem. 2010, 56, 327-329.

[12] Agency for Healthcare Research and Quality (AHRQ), Genomics test for ovarian cancer detection and management. AHRQ, Rockville, MD 2006.

[13] Zhang, Z., Chan, D. W., Cancer proteomics: in pursuit of "true" biomarker discovery. Cancer Epidemiol. Biomarkers Prev. 2005, 14, 2283-2286.

[14] Committee on Assessing Integrity in Research Environments, Integrity in scientific research: creating an en- vironment that promotes responsible conduct. National Academies Press, Washington, DC 2002.

[15] National Research Council, International regulatory harmonization amid globalization of drug development: workshop summary. National Academies Press, Washington, DC 2013.

[16] Hood, L., Systems biology and p4 medicine: past, present, and future. Rambam Maimonides Med. J. 2013, 4, e0012.

[17] Roychowdhury, S., lyer, M. K., Robinson, D. R., Lonigro, R. J. et al., Personalized oncology through integrative highthroughput sequencing: a pilot study. Sci. Transl. Med. 2011, 3, 111 ra121.

[18] Roychowdhury, S., Chinnaiyan, A. M., Advancing precision medicine for prostate cancer through genomics. J. Clin. Oncol. 2013, 31, 1866-1873.

[19] Hayes, D. F., Allen, J., Compton, C., Gustavsen, G. et al., Breaking a vicious cycle. Sci. Transl. Med. 2013, 5, 196cm196. 